\title{
Thermal Energy Storage Systems: Power-to-Heat Concepts in Solid Media Storage for High Storage Densities
}

\author{
Sergej Belik ${ }^{1}$, Volker Dreißigacker ${ }^{1}$, Mila Dieterich ${ }^{1}$ and Werner Kraft $^{2}$ \\ 1. German Aerospace Center (DLR), Institute of Engineering Thermodynamics, Stuttgart 70569, Germany; \\ 2. German Aerospace Center (DLR), Institute of Vehicle Concepts, Stuttgart 70569, Germany
}

\begin{abstract}
The German Aerospace Center has merged a wide range of technological research and development for future cars in a project called "Next Generation Car". Within this large research project, three vehicle concepts for different applications (urban, regional and interurban) and with different powertrains (fuel-cell, battery and hybrid) will be developed. Research questions on different levels from conceptual question about vehicle modularity down to detailed technological aspects like combining hydrogen storage with cabin climatization and a systematic investigation of different thermal energy storage systems for electric vehicles concepts are covered by this project. To the latter, the contribution shows an overview about three thermal storage technologies - sensible solid media, metallic latent and thermochemical thermal energy storage systems-and details about the development of an electrically heated (power-to-heat) solid media storage system to achieve high storage densities and to allow flexible thermal discharging values. Central works target the identification of suitable thermal management solutions in future electric vehicle concepts to increase range, efficiency and flexibility.
\end{abstract}

Key words: Energy management, thermal energy storage systems, power-to-heat, solid media storage, high thermal energy density.

\section{Introduction}

The project NGC (Next Generation Car) is a large scale project developing new EVs (electric vehicles) concepts and the related technologies. The project covers six research and development domains: the overall vehicle concepts, the lightweight body design, advanced powertrain, efficient energy management, mechatronic chassis and vehicle intelligence. The project has a holistic approach focusing on integrated development of technologies, methods and tools for future cars [1].

Within this project, systematic investigations of different thermal energy storage systems for electric vehicle concepts are conducted. Those technologies open up the potential to increase range and efficiency as well as improved flexibility in future mobility

Corresponding author: Sergej Belik, M.Eng., research fields: thermal energy storage, CAES, A-CAES, and power-to-heat technologies. E-mail: Sergej.Belik@dlr.de. concepts through innovative solutions in preheating vehicle components and in cabin climatization.

Major objectives include the identification of potential applications of thermal energy systems in various EV concepts, the determination of specifications and the development of suitable design options with high storage densities and thermal performances. Here, the investigated thermal energy systems include sensible solid media, metallic latent and thermochemical storage technologies, which enable - depending on the vehicle application and physical principle- high potentials to achieve the target values in future mobility concepts $[1,2]$.

The contribution outlines the current status of the development work on thermal energy storage systems for different vehicle applications and provides results for the dimensioning of electrically heated sensible solid media storage for cabin heating in EV concepts. 


\section{Thermal Energy Storage Systems for Future Electric Vehicle Concepts}

In future electric vehicle concepts, suitable thermal management solutions are needed to increase range, efficiency and flexibility [3,2]. One central element to achieve these objectives includes the integration of thermal energy storage systems. In times of waste heat, such a system stores thermal energy (charging mode) and provides thermal energy in times of demand (discharging mode). In the following, central technology characteristics and scientific works within the project NGC will be explained.

\subsection{Metallic Latent Thermal Energy Systems}

At latent thermal energy storage, the phase change of a material is used to store thermal energy. By the definition of the name, the conditions whereas the thermal energy is stored are given. As phase changes occur at nearly constant temperatures, thermal energy can be stored without changing the temperature of the material. As phase change, both the change from solid to liquid and the change from liquid to gaseous condition come in question for technical solutions. Common applications for latent thermal energy storage are, e.g., self-heating pads, thermal energy storages for solar power plants or house fronts. Typical materials used for these applications are salts, mixtures of salts, waxes or paraffins [3].
Current research at Institue of Vehicle Concepts focuses on thermal energy storage systems based on mPCM (metallic Phase Change Materials). With the use of mPCM both, the sensible and latent thermal energy is used to enable high energy densities (compare Fig. 1). As phase change, the conversion between solid and liquid is used. As quite generally known, the above mentioned conventional phase change materials suffer from their low thermal conductivities, limiting thermal energy storage applications regarding to high thermal heat transfer rates.

In contrast, mPCM show thermal conductivities of a magnitude of about 100 times higher with similar energy densities compared to conventional PCM. High charging and discharging rates can be realized to allow manifold working potentials in future electric vehicle concepts.

In the current NGC project, the identification of promising $\mathrm{mPCM}$ as well as their suitable housing materials are focused. Additionally, functional concepts and promising use cases for thermal energy storage systems using $\mathrm{mPCM}$ are derived from the requirements resulting out of the application in electric vehicles. As the main use case, electric pre-charging of the storage up to $600{ }^{\circ} \mathrm{C}$ at the loading station within a short time of 30 min maximum before driving is the most promising scenario. Thermal energy

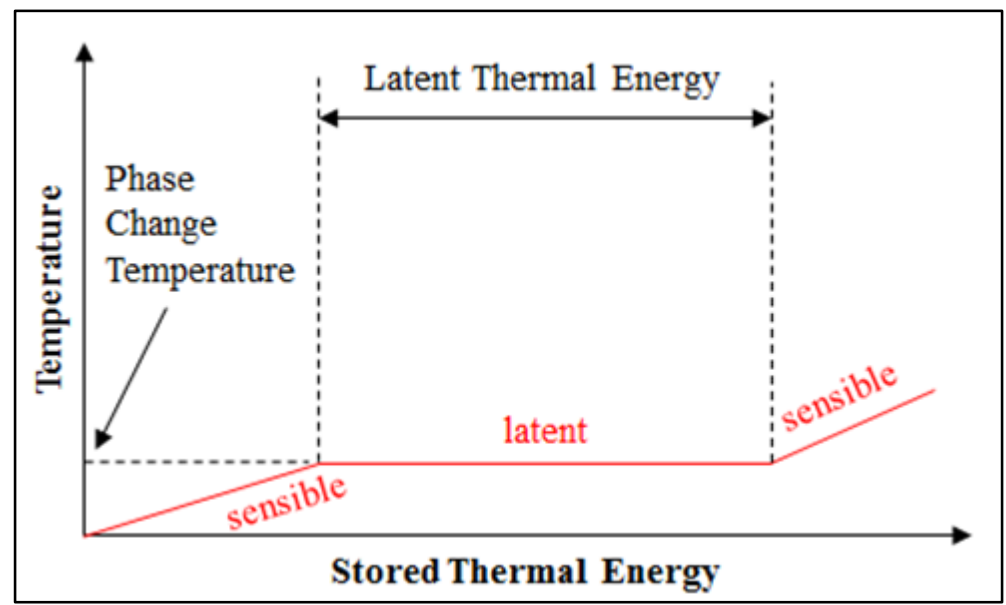

Fig. 1 Principle of the combination of sensible and latent thermal energy storage in $\mathrm{mPCM}$. 
stored like this can be used afterwards while driving for heating the vehicles interior and saving the energy of the traction battery to reduce range reduction. To fulfil the requirements of this scenario, current concepts use electric resistance heaters for charging the storage. Discharging is realized by perfusing a heat exchanger integrated into the storage with air, soaked in from the ambience. The regulation of the thermal output and the mass flow of the air supplied to the interior of the vehicle is implemented by mixing the hot air stream out of the storage with a cold air stream from the ambience.

\subsection{Thermochemical Energy Storage Systems}

Thermochemical energy storages store the reaction heat of a solid/gas reaction. Especially metal hydrides react very fast at comparably low temperature levels (-20 to $130{ }^{\circ} \mathrm{C}$ considered in this work) according to the following equation:

$$
\mathrm{M}+\frac{\mathrm{x}}{2} \mathrm{H}_{2} \Leftrightarrow \mathrm{MH}_{\mathrm{x}}+\Delta_{\mathrm{R}} \mathrm{H} .
$$

where, $\mathrm{M}$ is the metal; $\mathrm{MH}$ is the metal hydride and $\Delta_{\mathrm{R}} \mathrm{H}$ is reaction enthalpy. The temperature level at which the hydrogen is absorbed or desorbed depends on the pressure level of the hydrogen and vice versa. This relationship can be illustrated in a Van't
Hoff-plot, see Fig. 2, depicting the thermodynamic interaction between two different metal hydrides in a closed system.

The different materials are connected by a gas tube and can be separated by a valve. In the charged state of the thermal energy storage, metal hydride $B$ (MH_B) contains much hydrogen whereas metal hydride A ( $\left.\mathrm{MH}_{-} \mathrm{A}\right)$ contains only little hydrogen. Both are at ambient temperature, therefore the pressure of $\mathrm{MH}$ _B is higher than in $\mathrm{MH}$ _A. Once the valve is opened for discharging (indicated in blue in Fig. 2), hydrogen flows from $\mathrm{MH}_{-} \mathrm{B}$ to $\mathrm{MH}$ leading to desorption of more hydrogen in $\mathrm{MH} B$ which requires heat from the ambient and absorption of hydrogen in $\mathrm{MH}$ _A which releases heat. The temperature of the released heat in $\mathrm{MH}$ _A rises according to its equilibrium properties to the level of the preheating temperature. The storage is charged (indicated in orange in Fig. 2), with heat at charging temperature level, e.g., from electricity when the electric vehicle is charging, which is supplied to MH_A. The pressure rises according to the characteristics given in Fig. 2 and exceeds the pressure level in $\mathrm{MH}$ _B at ambient temperature. Now the valve is opened and hydrogen flows back into MH_B. The absorption heat is released to the ambient.

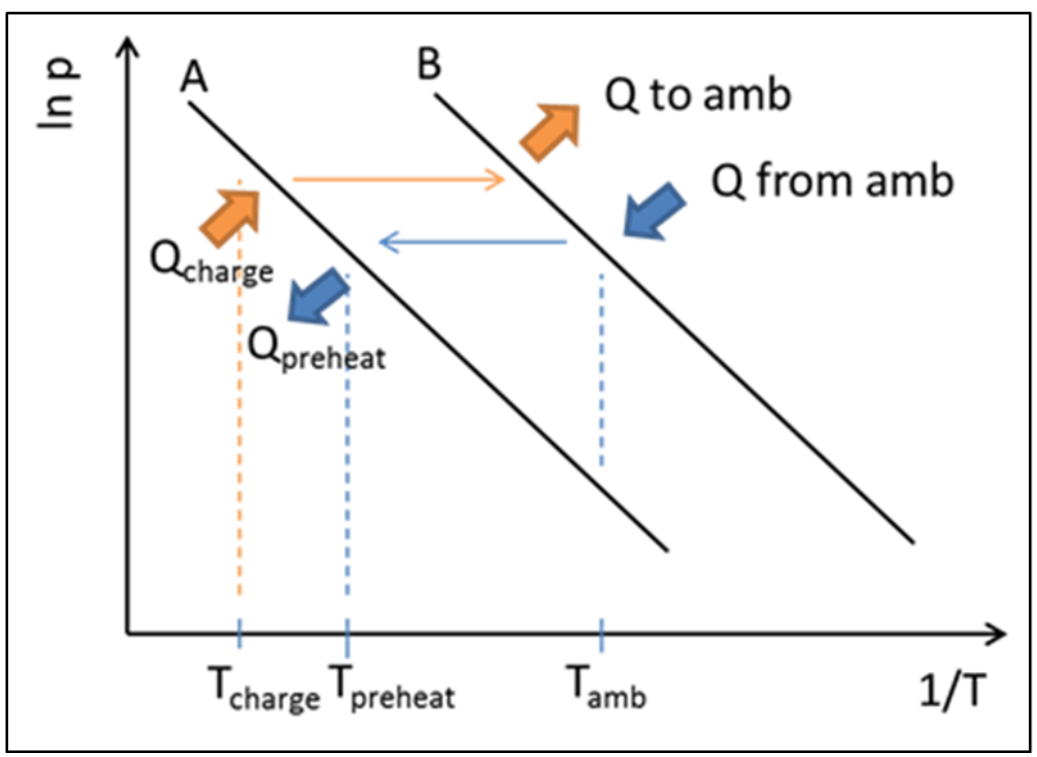

Fig. 2 Van't Hoff plot of two different metal hydrides A and B. 
After regeneration, the valve is closed and the energy is stored free of losses until it is needed again. Such closed thermochemical energy storage does not require or release hydrogen. The operation pressure depends on the chosen materials and was below 10 bar in this work. Also, no insulation is required for these storages.

Building heat, heat from electricity when charging or waste heat in the vehicle, even at low temperature levels, can be used to charge the energy storage. This energy can be used, e.g., at the next driving cycle to preheat vehicle components to shorten the cold start phase. This concept was already investigated in Ref. [4], also presented at the EVS30 [5] and will be further improved as part of the NGC project [1].

\subsection{Sensible Solid Media Thermal Energy Systems}

For applications with gaseous heat transfer media, sensible solid media thermal energy storages are a suitable solution. Installations of these regenerator-type thermal energy storage systems can be distinguished into moving and stationary beds. For fixed bed arrangements, the storage consists of stacks of bricks of various shapes and sizes. Depending on the required thermal characteristics, different types of bricks are used.

Where a substantial temperature decrease during discharging can be allowed, e.g., in steel and glass industries, checker bricks with small specific surfaces and small void fractions can be used. In contrast, applications requiring a low heat resistance, e.g., in RTO (regenerative thermal oxidizers) for the purification of air, honeycomb or saddle structures given in Fig. 3 are used.

Apart from their ability to make use of various materials, such inventory options allow various geometries with large heat transfer areas, thus an efficient thermal utilisation of the storage material, high thermal energy densities through storing of high temperature heat and excellent thermal dynamics due to the direct contact between the fluid and the storage inventory. In industrial applications, the thermal cyclic procedure consists of a charging and discharging period, where heat is transferred between the gaseous heat transfer media and the inventory options.

For applications in electric vehicle concepts, adaptations of this technology due to missing high

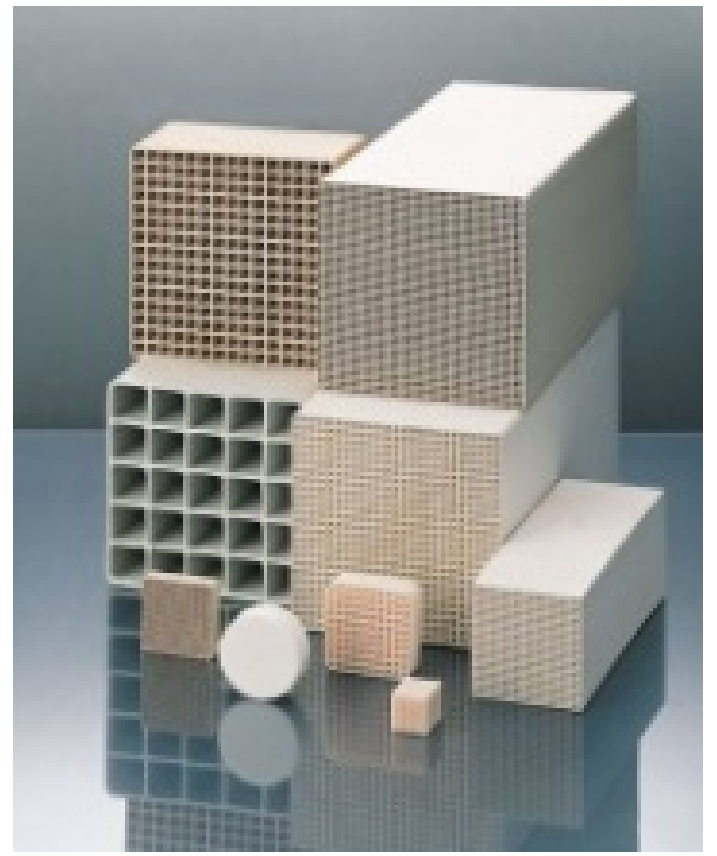

Fig. 3 Commercial solid media inventory options for industrial high temperature applications. 
temperature sources will be investigated inside the NGC project. Central development works include the integration of an electrical heating system inside the solid media to achieve high thermal storage densities, the elaboration of high efficient thermal insulation concepts, a bypass operation option during thermal discharging for a flexible heat supply with defined temperature conditions and electro-thermal simulation studies to identify suitable design solutions. As central element of this thermal storage technology, the power-to-heat concepts inside a suitable solid media inventory option will be investigated in detail. First results including the modelling approach for direct and indirect resistance heating systems as well as design calculations will be presented in the following.

\section{Power-to-Heat Concepts in Solid Media Sensible Thermal Energy Systems}

Hence in an electric driven vehicle, high-temperature energy sources are not available, the sensible TES (thermal energy storage) is heated by electric power during a vehicle halt (charging cycle) to temperatures of up to $700{ }^{\circ} \mathrm{C}$. As shown in Fig. 4, this high temperature heat is used during the drive (discharging cycle) to supply the vehicle cabin via a bypass system with conditioned air (here $60^{\circ} \mathrm{C}$ ).

This operation mode ensures high thermal energy densities due to the transformation from electric $\mathrm{P} 2 \mathrm{H}$ (power to heat) and flexible supply of conditioned air at various temperatures. As an example for reachable energy densities, Fig. 5 shows the effective gravimetric energy densities relating to electrical heating temperatures for an exemplary design with a required thermal capacity of 2.5 and $5 \mathrm{kWh}$, and a maximum surface temperature of thermal insulated containment of $60^{\circ} \mathrm{C}$. The thermal insulation concept is based on two layered set-up: a vacuum insulation with maximum operating temperature of $500{ }^{\circ} \mathrm{C}$ and a

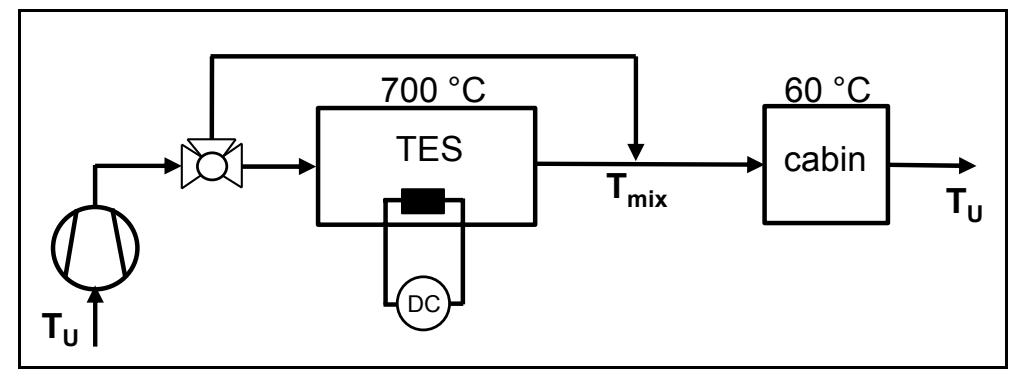

Fig. 4 P2H concept for air conditioning of vehicle cabin.

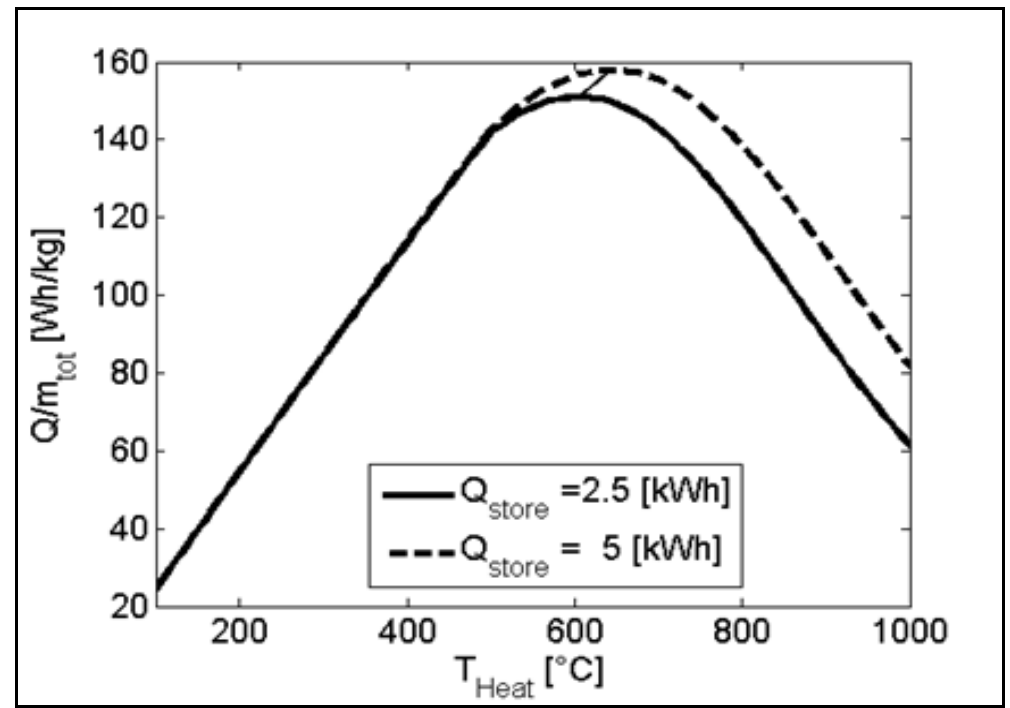

Fig. 5 Gravimetric energy density vs. heating temperature for 2.5 and $5 \mathrm{kWh}$ storage capacity. 


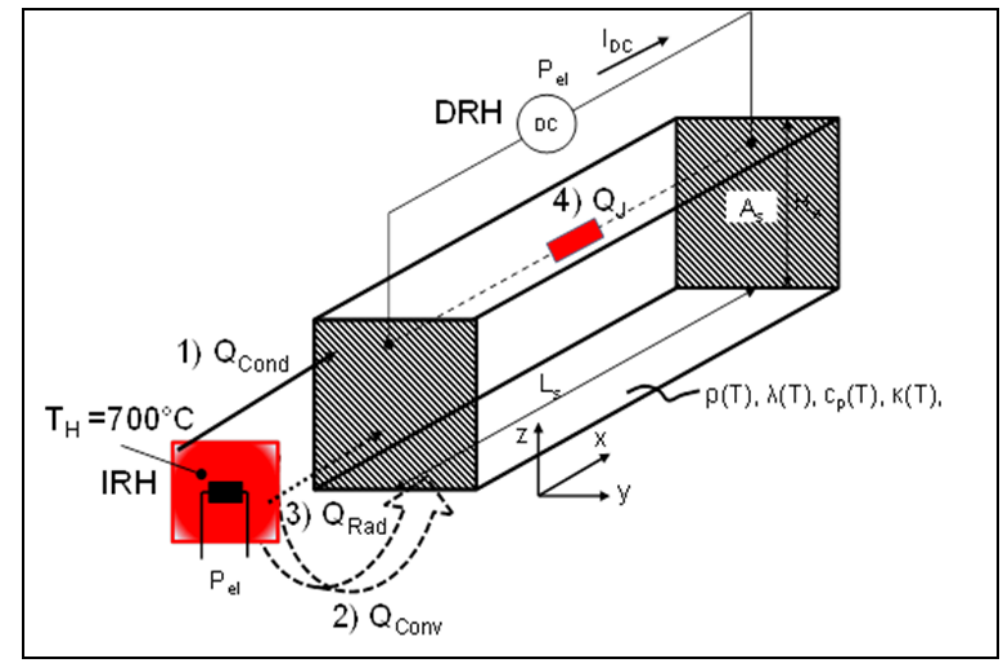

Fig. 6 Investigating concepts for $\mathrm{P} 2 \mathrm{H}$ in a solid material storage

microporous material for temperatures up to $1,000{ }^{\circ} \mathrm{C}$.

The appearing peak results from increasing energy density with higher electrical heating temperatures inside the solid medium and simultaneously increasing insulation demand to achieve the fixed surface temperature of $60{ }^{\circ} \mathrm{C}$.

Considering the technical realization of such a resistance heating system within a solid media storage, various electro-thermal concepts as well as operating strategies can be developed. With regard to existing, direct and indirect options of resistance heating, the following electro-thermal concepts are derived. In case of DRH (direct resistance heating), the solid media storage to be heated serves simultaneously as a resistor connected with a power supply within an electric circuit. Concerning IRH (indirect resistance heating), an external electric heater is used to heat up the solid media storage. In this process, the heat flow is transmitted by heat conduction, convection or radiation. Consequently, the heating concepts to be investigated are developed from these heat transfer mechanism as well as from Joule's heat caused by direct resistance heating inside the solid media. Thus, there are three $\mathrm{P} 2 \mathrm{H}$-concepts to be modelled for IRH and one for DRH, which are schematized in Fig. 6.

\section{Numerical Analysis}

The focus here is based on an accurate modelling of the derived electro-thermal concepts for comparing these with regard to heat flux rates. Besides that, these models can be used for wide-ranged simulation studies including material, geometry and process parameters to identify promising storage designs and to fulfill the target values (e.g., high energy density) for a selected electric vehicle application.

Due to high superficial areas, which are needed for an efficient heat transfer during the discharging cycle, a honeycomb design with a void fraction of $\varepsilon=0.25$ and a superficial area of $200 \mathrm{~m}^{2} / \mathrm{m}^{3}$ is chosen.

\subsection{Modelling Approach}

For modelling the heat transfer mechanism within the honeycomb solid media, a one-dimensional heat transfer analysis is performed considering different boundary conditions for each concept. Due to wide-ranged simulation studies, a time efficient approach is required without losing accuracy. Therefore, an approach is applied derived from porosity model [6] where the porosity $\varepsilon$ [-] is a ratio of air-filled volume to the total volume of the solid media. The thermos-physical properties as density $\rho$ $\left[\mathrm{kg} / \mathrm{m}^{3}\right]$, heat capacity $c_{p} \quad[\mathrm{~J} / \mathrm{kgK}]$ and thermal conductivity $\lambda[\mathrm{W} / \mathrm{mK}]$ are temperature dependent as well as the specific resistance $\kappa[\Omega \mathrm{m}]$ of investigated solid media [7]. 
$(1-\varepsilon) \rho c_{p} \frac{\partial T_{s}}{\partial t}=\lambda \frac{\partial^{2} T_{s}}{\partial x^{2}}+\kappa \frac{I_{D C}^{2}}{A_{s}^{2}}-k_{e f f} a_{v}\left(T_{s}-T_{\text {wall }}\right)(1)$

Concerning the heat losses via the containment surface, an averaged total heat transfer coefficient of $k_{\text {eff }}=0.011 \mathrm{~W} / \mathrm{m}^{2} \mathrm{~K}$ for the two layered thermal insulation design is applied in consideration of a volume specific containment area of $a_{v}=30 \mathrm{~m}^{2} / \mathrm{m}^{3}$, a maximum solid media temperature of $T_{s}=700{ }^{\circ} \mathrm{C}$ and an outer surface temperature of $T_{\text {wall }}=60{ }^{\circ} \mathrm{C}$. Calculating Joule's heat applied for the DRH-concept, the electric current $I D C$ [A] is calculated using Ohm's law with an applied voltage of $230 \mathrm{~V}$ and an ohmic resistance $R[\Omega]$ of the solid media calculated using the length $L_{s}[\mathrm{~m}]$ and the current carrying area $A_{s}\left[\mathrm{~m}^{2}\right]$ of the honeycomb:

$$
R=\kappa \frac{L_{s}}{A_{s}}
$$

For numerical analysis of elaborated IRH-concepts, the Joule's heat term is set to zero. In this case, the heat is transported over the system's border using boundary conditions.

\subsection{Boundary Conditions and Material Selection}

In order to calculate the heat propagation inside the honeycomb, the following boundary conditions for the IRH-concepts have been applied using symmetry conditions and the heating source with $T_{H}=700^{\circ} \mathrm{C}$ :

(1) Heat conduction:

$$
\begin{gathered}
-\lambda{\frac{\partial T_{s}}{\partial x_{x=0}}}^{=}=\frac{\lambda_{c o n}}{s_{c o n}}\left(T_{H}-T_{s, x=0}\right) \text { and } \\
-\lambda{\frac{\partial T_{s}}{\partial x_{x=L_{s}}}=0}^{=}
\end{gathered}
$$

where, $\lambda_{\text {con }}=3 \mathrm{~W} / \mathrm{mK}$ represents a heat-conducting paste with an assumed contact gap of $s_{c o n}=3 \mathrm{~mm}$ [8];

(2) Convection:

$$
\begin{gathered}
-\lambda{\frac{\partial T_{s}}{\partial x_{x=0}}}=\alpha_{H T F}\left(T_{H}-T_{s, x=0}\right) \text { and } \\
-\lambda{\frac{\partial T_{s}}{\partial x_{x=L_{s}}}=0}^{=}
\end{gathered}
$$

where, $\alpha_{H T F}=150 \mathrm{~W} / \mathrm{m}^{2} \mathrm{~K}$ is assumed for air as heat transfer fluid;

(3) Radiation:

$$
\begin{aligned}
-\lambda{\frac{\partial T_{s}}{\partial x_{x=0}}}^{=} & \frac{\sigma}{\left(\frac{1}{\varepsilon_{H}}+\frac{1}{\varepsilon_{S}}-1\right)}\left(T_{H}^{4}-T_{s, x=0}^{4}\right) \\
& -\lambda{\frac{\partial T_{s}}{\partial x_{x=L_{s}}}=0}=0
\end{aligned}
$$

where, $\sigma$ is the Stefan-Boltzmann constant and $\varepsilon_{H}=$ 0.85 as well as $\varepsilon_{s}=0.62$ is estimated emissivities for the heating source and the solid media.

For the DRH concept, an idealized electrical contacting is assumed. Therefore, the electrical contacting provides adiabatic conditions:

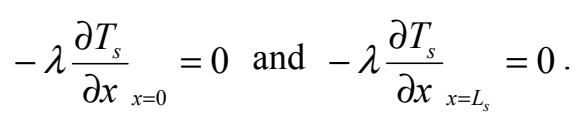

In a first step, silicon carbide (2H-SiC) with a hexagonal crystal structure and $70.0 \mathrm{Si}$ and $30.0 \mathrm{C}$ $\mathrm{wt} \%$ for DRH and the IRH concepts is chosen. This material option meets the requirements stated for thermal energy storage in electric vehicle applications and can be used as electroconductive material for the DRH concept. These are above all the thermal and electrical properties as high averaged values for heat capacity $(1,095 \mathrm{~J} / \mathrm{kgK})$, density $\left(2,960 \mathrm{~kg} / \mathrm{m}^{3}\right)$, thermal conductivity $(71.5 \mathrm{~W} / \mathrm{mK})$ and specific resistance $(41.05 \Omega \mathrm{m})$ shown in Fig. 7.

\section{Numerical Results}

In order to compare the identified concepts, boundary conditions derived from practice have been chosen. Thus, the IRH-concepts are heated with an external temperature of $700{ }^{\circ} \mathrm{C}$, the DRH-concept is supplied with a voltage of $230 \mathrm{~V}$. Furthermore, the utilization factors of the solid media storage after charging are varied in a range from 0.85 to 0.90 (complete heating with $700{ }^{\circ} \mathrm{C}$ corresponds to 1), where initial temperatures of $20^{\circ} \mathrm{C}$ are defined.

For fulfilling the target values of $2.5 \mathrm{kWh}$ charging capacity and heating times $\tau_{H}$ varied from 2 to $30 \mathrm{~min}$, iterative simulation runs including geometric data as length $L_{S}$ and heat transfer area $A_{S}$ are conducted. The 

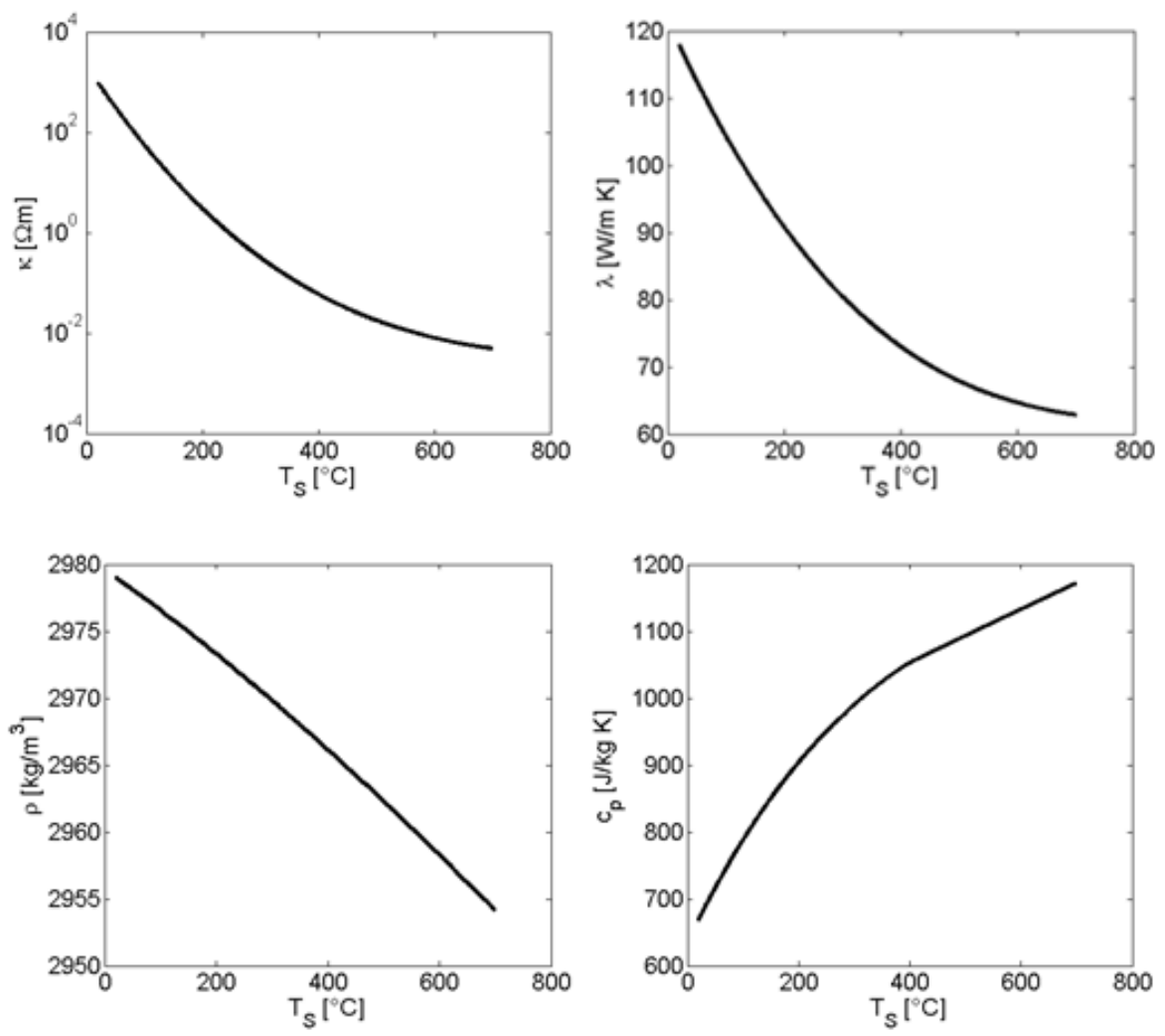

Fig. 7 Material properties of $2 \mathrm{H}-\mathrm{SiC}$ with $70.0 \mathrm{Si}$ and $30.0{ }^{\circ} \mathrm{C}$ wt\% [7].

range of results for each concept indicated in Fig. 8 occurs from varied utilization factors of the solid media storage. Thus greater values for average heat flux density are complying with the utilization factor of 0.85 due to smaller heat transfer areas needed to heat up the body in the same time. The decreasing characteristics related to heating time result from lower power needed to reach $2.5 \mathrm{kWh}$ in greater time.

The comparison shows high values for IRH-concepts, especially for the concept based on heat conduction. Reasons for that are in particular the lower thermal contact resistance which, in addition to high thermal conductivity of $2 \mathrm{H}-\mathrm{SiC}$, leads to better temperature propagation inside the solid media. On the other hand, the DRH-concept shows lower average heat flux densities over heating time, due to high specific resistance of chosen SiC. Thus, larger current carrying areas are needed to decrease the ohmic resistance $R$ in Eq. (2) in order to heat up the solid media with high electric currents.
High values for specific resistance occur especially at the beginning of the heating process where solid temperatures are less than $150{ }^{\circ} \mathrm{C}$, considering the logarithmic decrease of specific resistance in Fig. 7.

This high resistance limits the electric current for a fixed voltage indicated in Eq. (1), which is responsible for the heating due to Joule's effect. This limitation is demonstrated through the temperature rise in Fig. 9 (DRH) for an exemplary heating time of $20 \mathrm{~min}$ and an utilization factor of 0.9. Comparing to IRH-concepts, the averaged solid media temperature of DRH has much lower gradients up to a temperature of about $150{ }^{\circ} \mathrm{C}$. After that, the specific resistance decreases exponentially and the electric current is increasing, thus the solid media temperature. Accordingly, the specific resistance has a significant impact on performance of direct resistance heating process and needs to be reduced in range of $20 \sim 150{ }^{\circ} \mathrm{C}$ in order to reach higher heat flux densities for DRH-concept using $2 \mathrm{H}-\mathrm{SiC}$. On the other hand, 


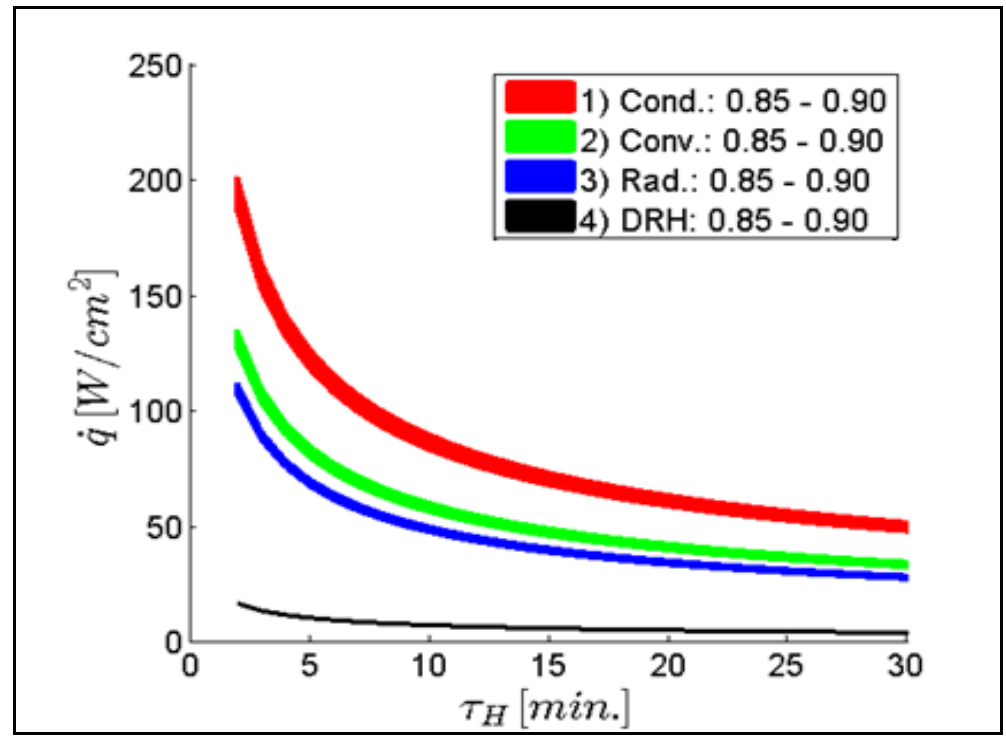

Fig. 8 Comparison of concepts: average heat flux density vs. heating time.

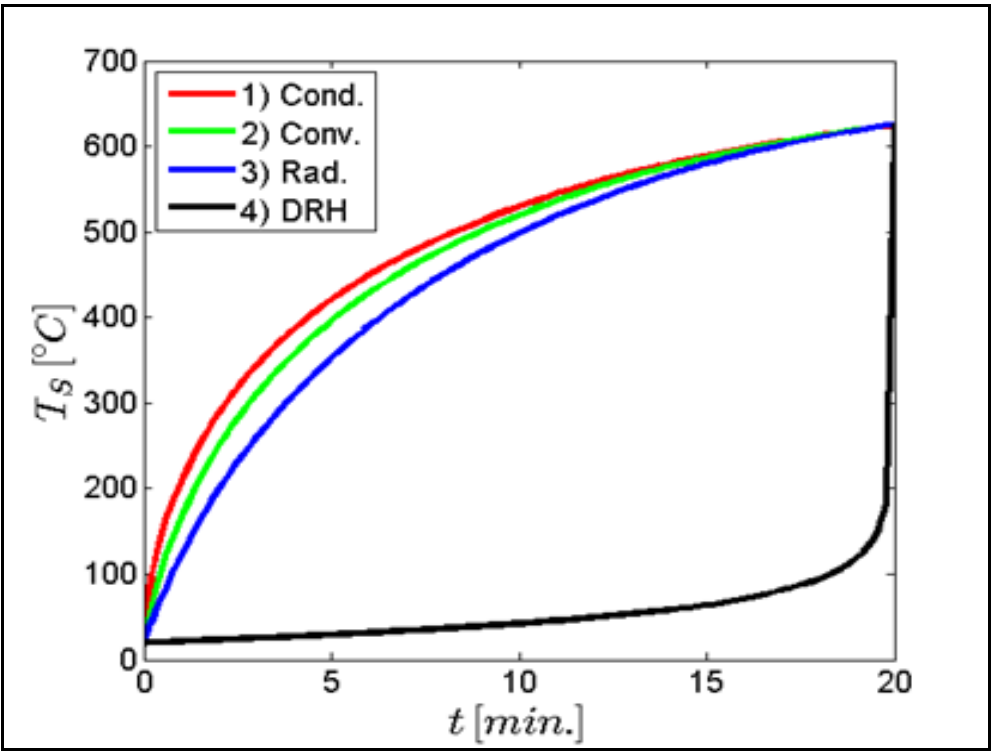

Fig. 9 Time characteristic of averaged solid media storage temperatures for $\tau_{H}=20 \mathrm{~min}$.

this value has to be limited at higher temperatures in order to prevent high electric currents within supply lines.

Due to prescribed target values, all four concepts reach gravimetric energy densities of $184 \mathrm{Wh} / \mathrm{kg}$ for a utilization factor of 0.9 and a storage mass of $13.4 \mathrm{~kg}$. Considering the system's extension by the two-layered insulation concept, effective energy densities of up to $160 \mathrm{Wh} / \mathrm{kg}$ are reachable.

Taking into account a charging time of $20 \mathrm{~min}$, a promising concept of heat conduction combined with honeycomb design has dimensions of $184 \times 184 \times 178$ $\mathrm{mm}$ to fulfil the prescribed target values.

\section{Conclusion}

Within the large research project "Next Generation Car", a systematic investigation of thermal energy storage systems for electric vehicle concepts is conducted. The potentials for such storage systems in future vehicle applications will be identified and elaborated for different storage technologies. First results for an electrically heated (power-to-heat) solid 
media storage system show high effective thermal storage densities of up to $160 \mathrm{Wh} / \mathrm{kg}(184 \mathrm{Wh} / \mathrm{kg}$ only for solid material) with a flexible supply of conditioned air at various temperatures due to the bypass operation. The comparison between four $\mathrm{P} 2 \mathrm{H}$ concepts considering direct and indirect resistance heating of a silicon carbide honeycomb with a hexagonal crystal structure $(2 \mathrm{H}-\mathrm{SiC})$ shows highest averaged heat flux densities for an indirect concept with thermal conductive heat transport. Lowest heat flux densities occur for directly electrical heated concept due to the limitation by high specific resistivity of $2 \mathrm{H}-\mathrm{SiC}$. Using lower average specific resistivity for another type of $\mathrm{SiC}$, which is suitably configured for the respective application, the direct resistance heating concept should provide significant improved results. Thus, further investigations will be performed on material identification and selection.

\section{References}

[1] Friedrich, H. E., and Schmid, S. A. 2016. "Vehicle Concepts for Tomorrow's Demand: A European Research Perspective." Presented at International Conference on Advanced Automotive Technology
(ICAT), Gwangju, Republic of Korea.

[2] Lajunen, A., Hadden, T., Hirmiz, R., Emadi, A. 2017. "Thermal Energy Storage for Increasing Heating Performance and Efficiency in Electric Vehicles." In Proceedings of Transportation Electrification Conference and Expo (ITEC), 291-312.

[3] Wang, M., Craig, T., Wolfe, E., LaClair, T. J., Gao, Z.-M., Levin, M., Demitroff, D., Shaikh, F. 2017. "Integration and Validation of a Thermal Energy Storage System for Electric Vehicle Cabin Heating." SAE Technical Paper 2017-01-0183.

[4] Dieterich, M., Bürger, I., and Linder, M. 2017. "Open and Closed Metal Hydride System for High Thermal Power Applications: Preheating Vehicle Components." Int. J. Hydrogen Energy 42 (16): 1-13.

[5] Dieterich, M., Bürger, I., and Linder, M. 2017. "Next Generation Car-Coupled Thermochemical Reactions for Preheating Vehicle Components." Presented at EVS30 Symposium 2017.

[6] Kere, A., Goetz, V., and Py, X. 2014. "Dynamic Behaviour of a Sensible-Heat Based Thermal Energy Storage." Energy Procedia 49: 830-9.

[7] Goldsmith, A., and Waterman, T. E. 1959. Saint-Gobain Advanced Ceramics Data Sheet. WADC Tech. report, ASTIA document No. 207905.

[8] Müller, D. 2014. "Wärmeleitprodukte. ” Accessed June 6, 2017. http://www.mueller-ahlhorn.com/fileadmin/Down loads/PDF/PDFDateien/TG_20033_de.pdf. (in German) 